\title{
MICOSES PULMONARES NA INFÂNCIA: uma pesquisa bibliográfica
}

\author{
Tamires Patrícia SOUZA ${ }^{1}$ \\ Rafaela SOUZA ${ }^{2}$
}

\begin{abstract}
${ }^{1}$ Enfermeira. Especialista em Saúde Pública- Ênfase em Estratégia de Saúde da Família. Mestranda em Ciências Pneumológicas pela Universidade Federal do Rio Grande do Sul (UFRGS). tamires_psouza@yahoo.com.

${ }^{2}$ Nutricionista. Residente em Sistema Público de Saúde - Ênfase em Atenção Básica pela Universidade Federal de Santa Maria (UFSM). rafa_nutri@yahoo.com.br.
\end{abstract}

Recebido em: 03/05/2015 - Aprovado em: 05/08/2015 - Disponibilizado em: 30/10/2015

\begin{abstract}
RESUMO: As micoses sistêmicas ou pulmonares apresentam diversas características em comum, entre elas: Distribuição geográfica limitada, com principal ocorrência nas Américas. O objetivo do estudo é analisar na literatura científica o que se tem publicado sobre micoses pulmonares na infância. A pesquisa se baseou em um levantamento bibliográfico nas bases de dados Scielo, PubMed e LILACS. Utilizaram-se como critérios de seleção: que fossem artigos científicos, que estivessem nos idiomas português, inglês e espanhol, com ano de publicação entre 1975 e 2014. Foram excluídos trabalhos sem resumo e que não estivessem disponíveis na íntegra. Da pesquisa sistematizada, encontraram-se 95 publicações. Foram selecionadas 29 que passaram por leitura flutuante. Destas, foram selecionados aqueles com relevância do tema, consistência do estudo e contribuição para o tema abordado. Conclui-se com o presente estudo que são inúmeras as infecções micóticas que podem afetar as crianças, porém, em relação à infecções pulmonares, os relatos são escassos. Tais relatos remetem ao fato de que essas infecções atingem as crianças principalmente em estado de imunodepressão, como nos casos de leucemia.

Palavras-chave: Micoses pulmonares da infância. Micoses de vias aéreas infantis. Infecções pulmonares da infância. Infecções de vias aéreas de pré-escolares. Infecções micóticas.
\end{abstract}

\section{MYCOSES PULMONARY CHILDHOOD: a literature search}

\begin{abstract}
The systemic or pulmonary mycoses have several features in common, including: limited geographical distribution, with major occurrence in the Americas. The objective of the study is to analyze the scientific literature that has been published about pulmonary mycoses in childhood. The research was based on a bibliographic survey of the SciELO, PubMed and LILACS databases. Were used as selection criteria: they were papers that were in Portuguese, English and Spanish, with year of publication between 1975 and 2014 were excluded without abstract works and that were not available in full. The systematic search, we found 95 publications and 29 who have undergone initial reading selected. Of these, we selected those with relevance of the topic, the study consistency and contribution to the topic covered. We conclude with this study that there are numerous fungal infections that can affect children, however, in relation to lung infections, reports are scarce. These reports refer to the fact that these infections among children especially in a state of immunosuppression, as in cases of leukemia.
\end{abstract}

Keywords: Pulmonary mycoses childhood. Mycoses of children's airways. Pulmonary childhood infections. Tract infections flights from preschool. Mycotic infections.

\section{INTRODUÇÃO}

As micoses sistêmicas ou pulmonares apresentam diversas características em comum, entre elas: Distribuição geográfica limitada, com principal ocorrência nas
Américas (exceto a criptococose)

(TRABULSI \& ALTERTHUM, 2008); Para os autores, os agentes etiológicos estão presentes no solo ou em dejetos animais e a principal porta de entrada são as vias aéreas superiores. 
A Resposta Celular a esses agentes (HEYDON et al., 2006), consiste em geral, em um processo granulomatoso, e depende da capacidade imunológica dos indivíduos afetados. As micoses podem ser responsáveis pelo adoecimento de adultos e crianças, sendo mais de $90 \%$ das infecções assintomáticas ou de rápida evolução. As micoses endêmicas nos EUA são responsáveis pela hospitalização de 4,6 crianças a cada 1 milhão de pessoas, e pela mortalidade de $5 \%$ das crianças imunocomprometidas (HEYDON et al., 2006).

Devido a sua importância, o objetivo do estudo é analisar na literatura científica o que se tem publicado sobre micoses pulmonares na infância

\section{METODOLOGIA}

Trata-se de um trabalho apresentado para a disciplina "Manifestações Pulmonares das Infecções por Fungos Oportunistas e Patógenos Primários", do curso de PósGraduação em Ciências Pneumológicas da Universidade Federal do Rio Grande do Sul (UFRGS).

Realizou-se uma pesquisa bibliográfica nas bases de dados Scielo, PubMed e LILACS, compreendendo trabalhos científicos. As palavras-chave utilizadas para a busca foram: micoses pulmonares da infância, micoses de vias aéreas infantis, infecções pulmonares da infância, infecções de vias aéreas de pré-escolares e infecções micóticas.

Utilizaram-se como critérios de seleção: que fossem artigos científicos, que estivessem nos idiomas português, inglês e espanhol, com ano de publicação entre 1975 e 2014. Foram excluídos trabalhos sem resumo e que não estivessem disponíveis na íntegra.

Da pesquisa sistematizada nas bases de dados, foram encontrados 95 publicações. Foram selecionadas 29 que passaram por leitura flutuante. Destes, foram selecionados aqueles com relevância do tema, consistência do estudo e contribuição para o tema abordado. Após a seleção da bibliografia, foram realizadas leituras exploratória e analítica, com o objetivo de descrever as formas nas quais as micoses pulmonares se manifestavam em crianças etiologia e possíveis tratamentos.

\section{RESULTADOS E DISCUSSÃO}

A seguir, serão apresentadas as principais micoses que acometem a saúde pulmonar na infância.

\section{Paracoccidioidomicose}

A paracoccidioidomicose, que também é conhecida como doença de Lutz ou blastomicose sul-americana, é causada pelo 
Paracoccidioides brasiliensis (BRUMMER et al., 1993). É endêmica em alguns países da América do Sul, ocorrendo grande número de casos no Brasil, Colômbia, Venezuela e Argentina. No Brasil, de acordo com Brummer et al. (1993), a paracoccidioidomicose incide, preferencialmente, em homens brancos, e a maioria dos casos ocorre na faixa etária entre 20 e 50 anos, sobretudo naqueles indivíduos que vivem em zona rural e que trabalham na lavoura, em contato duradouro com o solo, podendo também atingir crianças (BRUMMER et al., 1993).

O P. brasiliensis, segundo Wanke \& Aidé (2009), vive em solos férteis, úmidos, com abundante vegetação, podendo ser contaminante às pessoas ao inalarem pequenos fragmentos de hifas ou conídios na sua atividade diária na terra. As hifas ou os conídios ao chegarem aos alvéolos, iniciam a patogenia da doença, que é representada, inicialmente, por área de alveolite inespecífica (WANKE \& AIDÉ, 2009). De certo modo, de acordo com os autores, a instalação da doença ou não, se dá dependendo da quantidade de conídios inalados, do tempo de exposição e das condições imunológicas do indivíduo.

A paracoccidioidomicose pode se apresentar sob duas formas clínicas básicas: regressiva (primária) e progressiva (secundária) (WANKE \& AIDÉ, 2009). A forma regressiva resulta de um primeiro contato com o fungo em hospedeiros sem comprometimento imunológico, apresenta tendência à cura espontânea e é de evolução subclínica, às vezes, assintomática. A forma progressiva atinge indivíduos com alguma deficiência da imunidade e seria resultante, no adulto, de reativação da lesão primária quiescente, constituindo a forma clássica e mais comum da doença com manifestações pulmonares e extrapulmonares (DOMENICO et al., 2003).

A sintomatologia da paracoccidioidomicose é variável e na infância quase não apresenta sintomas respiratórios, sendo predominantes as lesões cutâneas, linfonodais nas cadeias cervicais, torácicas e abdominais, hepatoesplenomegalia e alterações intestinais (DOMENICO et al., 2003).

As alterações respiratórias principais, como tosse com expectoração mucoide e dispneia, de acordo com Domenico et al, (2003), estão frequentemente, associadas à lesão na pele e/ou mucosas da face e cavidade oral caracterizada como estomatite moriforme. Na fase mais avançada, o quadro assume as características clínicas dos processos supurativos broncopulmonares crônicos sem nenhuma especificidade, confundindo-se, na maioria das vezes, com a tuberculose (DOMENICO et al., 2003).

O diagnóstico do Paracoccidioides brasiliensis é baseado no exame microscópico direto da amostra com pus, escarro etc., onde podem ser visualizados: células 
leveduriformes, fungos esféricos ou ovais com dupla membrana e paredes espessas e refringentes, com três ou mais brotamentos (FONSECA et al., 1999). Além do exame microscópico do material coletado da lesão, também há testes sorológicos aos quais se pode recorrer na ausência desse material, com a reação de fixação do complemento e a precipitação em gel de ágar. O tratamento é feito de acordo com a forma clínica e estado imunológico do paciente, com: sulfamidas com ou sem trimetoprima; anfotericina B; miconazol e itraconazol (FONSECA et al., 1999).

\section{Histoplasmose}

A infecção humana por Histoplasma capsulatum var. capsulatum (H. capsulatum) é benigna e regressiva (GOODWIN et al., 1981). As manifestações clínicas da doença dependem do estado imunológico e anatomia do hospedeiro, bem como da concentração de propágulos fúngicos inalados. A deficiência de imunidade celular predispõe à doença disseminada e potencialmente fatal, se não tratada. $\mathrm{O}$ desarranjo anatômico do parênquima pulmonar propicia a colonização dos espaços aéreos em uma exposição prolongada ao fungo, e por mecanismo alérgico, desencadeia a doença crônica, acarretando fibrose pulmonar (KWONCHUNG \& BENNETT, 1992). Por outro lado, em um hospedeiro sadio, somente uma inalação maciça de propágulos fúngicos desencadeia a histoplasmose pulmonar aguda (GOODWIN \& DES PREZ, 1978).

$\mathrm{Na}$ histoplasmose pulmonar aguda, a febre, a mialgia e a hepatoesplenomegalia estão sempre presentes, geralmente acompanhadas de manifestações respiratórias como tosse seca, dor retroesternal, dispneia e, ocasionalmente, dor pleural. Radiologicamente, esta forma caracteriza-se por pequenas áreas de pneumonite em meio a infiltrado intersticial difuso, além de adenomegalia hilar. $\mathrm{Na}$ histoplasmose pulmonar crônica, as manifestações clínicas, as lesões radiológicas e a evolução são praticamente indistinguíveis da tuberculose pulmonar de reinfecção do adulto.

O diagnóstico definitivo é obtido laboratorialmente através de técnicas micológicas, histopatológicas e imunológicas (AIDÉ, 2009). De acordo com o autor, o cultivo é procedimento obrigatório no diagnóstico da enfermidade, principalmente nas formas disseminadas e na forma pulmonar crônica, onde pode apresentar sensibilidade acima de $85 \%$. Na histoplasmose pulmonar aguda, a regressão espontânea é regra, já que a doença, na maioria das vezes, é benigna e autolimitada, não necessitando de tratamento específico; sendo, contudo, essa conduta controversa (AIDÉ, 2009). Os principais medicamentos utilizados são os derivados imidazólicos, notadamente o itraconazol na dose de $200 \mathrm{mg}$ de $12 / 12 \mathrm{~h}$ por um período 
mínimo de 6 semanas. Anfotericina B é excelente opção terapêutica nas formas disseminadas (AIDÉ, 2009).

Em 2005 foi publicado um estudo descrevendo casos de Histoplasmose pulmonar aguda no Rio Grande do Sul, onde foram revistos 212 prontuários clínicos de pacientes com histoplasmose que constam nos arquivos do laboratório num período de 25 anos (1977 a 2002). Dezoito apresentaram quadro de histoplasmose pulmonar aguda (8,5\%). A idade variou de 8 a 63 anos (média de 35,4; mediana de 34,5). Doze pacientes eram do sexo masculino (67\%) (UNIS et al., 2005).

\section{Criptococose}

A criptococose é causada pelo fungo $C$. neoformans var. neoformans e C. neoformans var. gattii. A variedade neoformans é relacionada à excretas e hábitats de aves, principalmente pombos e periquitos (DARZÉ et al., 2000). De acordo com Darzé et al. (2000), a micose é de frequência elevada nas grandes cidades, onde são diagnosticadas suas formas clínicas mais graves. As formas subclínicas ou as que se manifestam como infecção respiratória inespecífica devem ser bastante frequentes (DARZÉ et al., 2000. Pode ser caracterizada como um agente oportunista, acometendo principalmente indivíduos imunocomprometidos. A variedade gattii pode ser encontrada juntamente com material orgânico em decomposição,

acometendo predominantemente indivíduos aparentemente normais, sobretudo adultos jovens de ambos os sexos e crianças, nativos de áreas tropicais e subtropicais (DARZÉ et al., 2000). A micose é adquirida após inalação de conídios infectantes que, chegam aos alvéolos e se multiplicam.

A criptococose apresenta-se clinicamente sob três formas: pulmonar regressiva, pulmonar progressiva e disseminada. A forma pulmonar regressiva, referida por Passoni et al. (1998), é assintomática e geralmente passa despercebida, sendo o diagnóstico feito pela análise histopatológica de nódulos pulmonares residuais. A forma pulmonar progressiva manifesta-se de forma insidiosa, podendo ser confundida com pneumonia de evolução crônica; radiologicamente, pode apresentar-se como massa periférica, de limites bem definidos, podendo ser confundida com neoplasia. $\mathrm{Na}$ forma disseminada, vários órgãos podem ser concomitantemente atingidos, notadamente o sistema nervoso central, determinando quadros neurológicos variados entre os quais meningoencefalite (PASSONI et al., 1998).

A doença é endêmica no Norte e no Nordeste do país, acometendo crianças, jovens e adultos, de ambos os sexos e todas as faixas etárias, com ou sem depressão do sistema imunológico (PASSONI et al., 1998). 
No Pará, no ano de 1999 foram registrados 19 casos em crianças com idade entre 3 a 12 anos, sendo que destes, 9 (47\%) causados por C. gatti. Houve óbito em $56,6 \%$ dos casos, complicações como cegueira em 2 pacientes e atrofia cortical em um paciente (FONSECA $e t$ al., 1999).

\section{Sarcoidose}

A sarcoidose é uma doença sistêmica, com comprometimento predominantemente pulmonar em adultos e crianças maiores, caracterizada pela formação de granulomas podendo ser confundida com tuberculose (CORRÊA et al., 1999). O quadro clinico da doença pode variar entre adultos e crianças, sendo frequentemente não diagnosticada nas crianças. A doença não tem sua etiologia esclarecida e, portanto, sua definição é basicamente clínico-patológica.

Nos EUA, a frequência estimada da doença em crianças é de 1/20 da encontrada em adultos (3\%-5\% dos casos). Entretanto, em países onde estudos radiológicos em massa da população são realizados, como a Hungria e o Japão, a prevalência em crianças parece ser a mesma que a em adultos (DONATO et al., 1991).

Para o autor, a sarcoidose, na população pediátrica, é mais frequentemente diagnosticada em adolescentes do que em crianças menores. Em crianças, os órgãos mais acometidos são os pulmões, linfonodos, olhos, pele, fígado e baço. Numa série de 27 casos, 29,6\% das crianças apresentaram comprometimento de três ou mais órgãos na apresentação. Os sintomas clínicos da sarcoidose na população pediátrica dependem da idade, sendo possível dividir as crianças em grupos de acordo com a idade: crianças até quatro anos, crianças com quatro a oito anos e crianças que, como o caso, são maiores do que oito anos de idade. Nestas, o quadro se assemelha bastante ao de adultos (DONATO et al., 1991).

Os sintomas respiratórios mais comuns são tosse e dispneia, podendo eventualmente ocorrer dor torácica. O exame físico pode evidenciar crepitações, roncos, sibilos e diminuição do murmúrio vesicular. A linfoadenopatia periférica é a anormalidade ao exame mais comum em crianças com sarcoidose e tipicamente consiste de nódulos firmes, indolores e móveis, como os encontrados (RIZZATO, 1998). Por sua vez, hepatoesplenomegalia ocorre em até $43 \%$ das crianças com sarcoidose em algum momento durante o curso clínico. A hipercalcemia ocorre em cerca de $30 \%$ das crianças e é geralmente assintomática, assim como a hipercalciúria associada. Entretanto, o efeito tóxico do cálcio nos túbulos renais pode produzir sintomas de poliúria, polidipsia e desidratação (RIZZATO, 1998).

O prognóstico da sarcoidose na população pediátrica, em longo prazo, parece não ser significativamente diferente do em 
adultos, sendo em geral favorável (MARCILLE et al., 1992). A maioria das crianças apresenta melhora considerável nos sintomas, nos achados radiológicos e em provas de função pulmonar. Porém, existem dados sugestivos de que o início da doença antes dos quatro anos de idade pode ser um indicador de mau prognóstico. Outras sequelas comuns são cegueira e paralisia facial (MARCILLE et al., 1992).

$\mathrm{O}$ tratamento em crianças, prednisona ou prednisona são geralmente iniciadas na dose de $1 \mathrm{mg} / \mathrm{kg} /$ dia. Uma redução gradual da dose é realizada, até uma dose de manutenção de $15 \mathrm{mg}$ de prednisona em dias alternados. A duração mínima do tratamento é de seis meses. Se um relapso ocorre, a dose é então novamente aumentada para a quantidade necessária para controlar as manifestações clínicas da doença e novamente reduzida para a dose de manutenção, que provavelmente será maior do que aquela usada quando do relapso (CHADELAT et al., 1993).

\section{Aspergilose}

Aspergillus spp. é um fungo encontrado em vários locais da natureza, sendo a fonte de contágio mais comum, as vias aéreas, acometendo gravemente pacientes imunodeprimidos, tanto crianças quanto adultos (WALSH et al., 2008). O A. fumigatus é o agente mais comum das várias formas de manifestação da aspergilose
(WALSH et al., 2008), porém, também podem ser encontrados casos de aspergilose por A. flavus, A. niger e A. terreus, sendo esse último resistente à anfotericina (BARNES \& MARR, 2006). Existem evidências de asma causada por Aspergillus spp. e da associação desse organismo com asma grave e letal (BARNES \& MARR, 2006).

As alterações pulmonares causadas pelo Aspergillus são variadas e dependentes do estado imunológico e das condições pulmonares do paciente. Reação de hipersensibilidade à inalação de esporos pode causar alveolite alérgica aguda, e colonização brônquica pode causar aspergilose broncopulmonar alérgica. Esta última é encontrada especialmente em pacientes com asma ou fibrose cística (WALSH et al., 2008). Caracteriza-se por episódios de asma aguda responsiva a corticosteroide ou por asma corticoide dependente, com sintomas não usuais de febre e hemoptise, com destruição da via aérea. Se tratada inadequadamente, o dano pulmonar permanente progride para fibrose (BARNES \& MARR, 2006).

Micetomas se desenvolvem por colonização secundária de cavidades pulmonares pré-existentes. Aspergilose invasiva e semi-invasiva afetam particularmente pacientes com estado imunológico alterado. A aspergilose broncopulmonar alérgica constitui uma forma de hipersensibilidade pulmonar associada à 
destruição das vias aéreas em resposta ao Aspergillus spp (AQUINO et al., 1994).

Para o diagnóstico, utilizam-se de critérios como a obstrução brônquica episódica, a eosinofilia periférica, a presença de anticorpos específicos contra antígenos de Aspergillus spp., a reação cutânea imediata a antígeno de Aspergillus spp., a elevação sérica de IgE, a presença de infiltrados pulmonares que resolvem com corticoides e bronquiectasias centrais. Os critérios diagnósticos secundários são: detecção de Aspergillus spp. em espécimes respiratórios, expectoração de moldes brônquicos, elevação de IgE específica contra Aspergillus spp. e reação cutânea tardia (WALSH et al., 2008).

Para estabelecer-se um diagnóstico provável, é necessário o preenchimento de pelo menos três critérios, como fatores de risco do hospedeiro; manifestações clínicas e radiológicas; e evidência microbiológica (cultura/antigenemia). Em virtude do potencial de progressão da doença, recomenda-se o tratamento precoce nos casos altamente suspeitos, enquanto a avaliação diagnóstica é conduzida. A monitorização da resposta terapêutica inclui a avaliação dos sintomas e sinais, assim como dos aspectos radiológicos, em intervalos regulares. $\mathrm{O}$ aumento progressivo da antigenemia significa mau prognóstico; porém, a normalização da mesma não pode ser utilizada como critério único para cessar o tratamento (WALSH et al., 2008).

\section{CONCLUSÃO}

Conclui-se com o presente estudo que são inúmeras as infecções micóticas que podem afetar as crianças, porém, em relação à infecções pulmonares, os relatos são escassos. Tais relatos remetem ao fato de que essas infecções atingem as crianças principalmente em estado de imunodepressão, como nos casos de leucemia. E, por outro lado, a maioria das infecções micóticas em crianças, passa despercebida, pois normalmente não apresentam sintomatologia característica em adultos. Provavelmente existam ainda inúmeras infecções micóticas que são responsáveis pelo adoecimento de crianças, todavia, procurou-se trazer de forma sucinta os relatos encontrados de infecções pulmonares em crianças, que apresentaram sintomas ou que foram diagnosticadas, e assim transformaram-se em relato de caso.

\section{REFERÊNCIAS}

AIDÉ, M.A. Curso de Atualização: Micoses. Histoplasmose. J Bras Pneumologia, 35(9):1145-51, 2009.

AQUINO, S.L.; KEE, S.T.; WARNOCK, M.L.; GAMSU. G. Pulmonary aspergillosis: imaging findings with pathologic correlation. AJR; 163:811-5, 1994.

BARNES, P.D.; MARR, K.A. Aspergillosis: spectrum of disease, diagnosis, and treatment. Infect Dis Clin North Am;20(3):545-61, vi, 2006. 
BRUMMER, E.; CASTAÑEDA, E.; RESTREPO, A. Paracoccidioidomycosis: an update. Clin Microbiol Rev; 6:89-117, 1993.

CHADELAT, K.; BACULARD, A.; GRIMFELD, A.; TOURNIER, G.; BOULE, M.; BOCCON-GIBOD, L.; CLEMENT, A. Pulmonary sarcoidosis in children: serial evaluation of bronchoalveolar lavage cells during corticosteroids treatment. Pediatr Pulmonol; 16:41-47, 1993.

CORRÊA, M.P.S.C.; OLIVEIRA, E.C.; DUARTE, R.R.B.S.D.; PARDAL, P.P.O.; OLIVEIRA, F.M.; SEVERO, L.C.

Criptococose em crianças no Estado do Pará, Brasil. Revista da Sociedade Brasileira de Medicina Tropical. 32(5):505-508, set-out, 1999.

DARZÉ, C.; LUCENA, R.; GOMES, I.; MELO, A. Características clínicas e laboratoriais de 104 casos de meningite criptocócica. Rev Soc Bras Med Trop; 33(1):21-6, 2000.

DOMENICO, C.; MOGAMI, R.; MIYAGUI, T. Tomografia Computadorizada de Alta Resolução nas Doenças Difusas Pulmonares: Correlação Anatomopatológica. 1a ed. São Paulo- Rio de Janeiro - Ribeirão Preto - Belo Horizonte: Atheneu 2003. 360 p.

DONATO, L.; BACULARD, A.; BOULE, M.; BOCCON-GIBOD, L.; GRINFELD, A.; TORNIER, G. Mediastino-pulmonary sarcoidosis in children. Clinical study, analysis of data of bronchoalveolar fluid lavage and respiratory function tests, therapeutic trends. Arch Fr Pediatr; 48:535$542,1991$.

FONSECA, E.R.S.; PARDAL, P.P.O.; SEVERO, L.C. Paracoccidioidomicose em crianças em Belém do Pará. Revista da Sociedade Brasileira de Medicina Tropical 32(1):31-33, jan-fev, 1999.

GOODWIN, R.A.; DES PREZ, R.M.

Histoplasmosis, state of the art. Am Rev Respir Dis;117:929-56, 1978.

GOODWIN, R.A.; LOYD, J.E.; DES PREZ,

R.M. Histoplasmosis in normal hosts.

Medicine (Baltimore); 60:231-66, 1981.
HEYDON, K.; WALSH, T.J.; THEOKLIS, A.; ZAOUTIS, E. Hospitalizations for Endemic Mycoses: A Population-Based National Study Jaclyn H. Chu, 1,2 Chris Feudtner,2,3,4,5 Clinical Infectious Diseases; 42:822-5, 2006.

KWON-CHUNG, K.J.; BENNETT, J.E. Histoplasmosis. In: KWON-CHUNG, K.J.; BENNETT, J.E. Medical mycology. Philadelphia: Lea \& Febiger; p.248-79, 1992.

MARCILLE, R.; MCCARTHY, M.; BARTON, J.W.; MERTEN, D.F.; SPOCK, A. Long term outcome of pediatric sarcoidosis with emphasis on pulmonary status. Chest;102:1444-1449, 1992.

PASSONI, L.F.C.; WANKE, B.; NISHIKAWA, M.M.; LAZERA, M.S. Cryptococcus neoformans isolated from human dwellings in Rio de Janeiro, Brazil: an analysis of the domestic environment of AIDS patients with and without cryptococcosis. Med Micol; 36:305-11, 1998.

RIZZATO, G. Clinical impact of bone and calcium metabolism changes in sarcoidosis. Thorax;53:425-429, 1998.

TRABULSI, L.R.; ALTERTHUM, F.

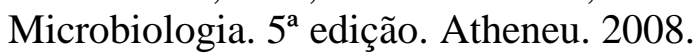
UNIS, G.; ROESCH, E.W.; SEVERO, L.C. Histoplasmose pulmonar aguda no Rio Grande do Sul. Jornal Brasileiro de Pneumologia 31(1) - Jan/Fev de 2005.

WALSH, T.J.; ANAISSIE, E.J.; DENNING, D.W.; HERBRECHT, R.; KONTOYIANNIS, D.P.; MARR, K.A.; et al. Treatment of aspergillosis: clinical practice guidelines of the Infectious Diseases Society of America. Clin Infect Dis;46(3):327-60, 2008.

WANKE, B.; AIDÉ, M.A. Curso de Atualização. Micoses. Paracoccidioidomicose. J Bras Pneumol; 35(9):1245-9, 2009. 